\title{
Mild solution of the heat equation with a general stochastic measure
}

\author{
by \\ VADYM RADCHENKO (Kyiv)
}

\begin{abstract}
The stochastic heat equation on $[0, T] \times \mathbb{R}$ driven by a general stochastic measure is investigated. Existence and uniqueness of the solution is established. Hölder regularity of the solution in time and space variables is proved.
\end{abstract}

1. Introduction. In this paper we consider the stochastic heat equation, which can formally be written as

$$
\left\{\begin{array}{l}
d u(t, x)=a^{2} \frac{\partial^{2} u(t, x)}{\partial x^{2}} d t+f(t, x, u(t, x)) d t+\sigma(t, x) d \mu(x), \\
u(0, x)=u_{0}(x),
\end{array}\right.
$$

where $(t, x) \in[0, T] \times \mathbb{R}, a \in \mathbb{R}, a \neq 0$, and $\mu$ is a stochastic measure defined on the Borel $\sigma$-algebra of $\mathbb{R}$. For $\mu$ we assume $\sigma$-additivity in probability only; the assumptions on $f, \sigma$ and $u_{0}$ are given in Section 4. We consider mild solutions to the formal equation (1.1) (see (4.1) below). We prove the existence and uniqueness of solution and obtain Hölder regularity of its paths.

The Gaussian white noise driven parabolic stochastic partial differential equations (SPDEs) were introduced and discussed initially in [21]. SPDEs with Poisson white noise were considered, for example, in [1]. In [4] SPDEs were studied as stochastic equations in function spaces. Hölder regularity of solutions of SPDEs of different types was considered in [3], [5], [9], [18], [21, Chapter 3]. In these and many other papers the stochastic noise satisfies some special conditions on distributions and moment existence, or has martingale properties. In this paper, we consider a very general class of possible $\mu$. On the other hand, the stochastic term in (1.1) is independent of $u$. A reason is that an appropriate definition of integral of a random function with respect to $\mu$ does not exist.

2000 Mathematics Subject Classification: 60H15, 60G17, 60G57.

Key words and phrases: stochastic heat equation, stochastic measure, Hölder regularity, Besov space. 
The equation (1.1) describes the evolution in time of the density $u$ of some quantity such as heat or chemical concentration in a system with random sources. The aim of the paper is to show that we can obtain and study the mild solution under some general conditions on the stochastic part of the equation.

The paper is organized as follows. In Section 2 we gather some basic facts about stochastic measures; in Section 3 two auxiliary lemmas for stochastic integrals are proved. A precise formulation of the problem and our assumptions are given in Section 4. Hölder continuity of the stochastic integral from our equation is proved in Sections $5-6$. Section 7 contains the main result of the paper.

2. Preliminaries. Let $L_{0}=L_{0}(\Omega, \mathcal{F}, \mathrm{P})$ be the set of all (equivalence classes of) real-valued random variables defined on a complete probability space $(\Omega, \mathcal{F}, \mathrm{P})$. Convergence in $L_{0}$ means convergence in probability. Let $\mathrm{X}$ be an arbitrary set and $\mathcal{B}$ be a $\sigma$-algebra of subsets of $\mathrm{X}$.

Definition. Any $\sigma$-additive mapping $\mu: \mathcal{B} \rightarrow L_{0}$ is called a stochastic measure.

In other words, $\mu$ is a vector measure with values in $L_{0}$. We do not assume positivity or moment existence for stochastic measures. In [7] such a $\mu$ is called a general stochastic measure. In the following, $\mu$ always denotes a stochastic measure.

Examples of stochastic measures are the following.

Let $\mathrm{X}=[0, T] \subset \mathbb{R}_{+}, \mathcal{B}$ be the $\sigma$-algebra of Borel subsets of $[0, T]$, and $X(t)$ be a continuous square integrable martingale. Then $\mu(A)=\int_{0}^{T} \mathbf{1}_{A}(t) d X(t)$ is a stochastic measure on $\mathcal{B}$. If $W^{H}(t)$ is a fractional Brownian motion with Hurst index $H>1 / 2$ and $f:[0, T] \rightarrow \mathbb{R}$ is a bounded measurable function then $\mu(A)=\int_{0}^{T} f(t) \mathbf{1}_{A}(t) d W^{H}(t)$ is also a stochastic measure on $\mathcal{B}$, as follows from [8, Theorem 1.1]. Other examples may be found in [7, Subsection 7.2]. Theorem 8.3.1 of [7] states conditions under which a process with independent increments generates a stochastic measure.

For deterministic measurable functions $g: X \rightarrow \mathbb{R}$, an integral of the form $\int_{\mathbf{X}} g d \mu$ is studied in [12] (see also [7, Chapter 7], [2]). The construction of this integral is standard, uses an approximation by simple functions and is based on results of $[16,17,19,20]$. In particular, every bounded measurable $g$ is integrable with respect to any $\mu$. An analogue of the Lebesgue dominated convergence theorem holds for this integral (see [7, Proposition 7.1.1] or [12, Corollary 1.2]). Path regularity of stochastic measures and parameter integrals with respect to $\mu$ are studied in $[13,14]$. Weak solutions of some SPDEs with stochastic measures were obtained in [15]. 
3. Properties of the integral with respect to stochastic measure. The following statement is a generalization of [14, Lemma].

Lemma 3.1. Let $f_{l}: X \rightarrow \mathbb{R}, l \geq 1$, be measurable functions such that $\bar{f}(x)=\sum_{l=1}^{\infty}\left|f_{l}(x)\right|$ is integrable with respect to $\mu$. Then

$$
\sum_{l=1}^{\infty}\left(\int_{\mathrm{X}} f_{l} d \mu\right)^{2}<\infty \quad \text { a.s. }
$$

Proof. Define $\xi_{l}(\omega)=\int_{X} f_{l} d \mu$. Suppose the lemma were false. Then

$$
\exists \varepsilon_{0}>0 \forall M>0 \exists m \geq 1: \mathrm{P}\left(\Omega_{m, M}=\left\{\omega \in \Omega: \sum_{l=1}^{m} \xi_{l}^{2}(\omega) \geq M\right\}\right) \geq \varepsilon_{0} .
$$

Consider independent Bernoulli random variables $\varepsilon_{l}, 1 \leq l \leq m$, defined on some other probability space $\left(\Omega^{\prime}, \mathcal{F}^{\prime}, \mathrm{P}^{\prime}\right), \mathrm{P}^{\prime}\left(\varepsilon_{l}=1\right)=\mathrm{P}^{\prime}\left(\varepsilon_{l}=-1\right)=1 / 2$. The following is a consequence of the Paley-Zygmund inequality:

$$
\mathrm{P}^{\prime}\left[\left(\sum_{l=1}^{m} \lambda_{l} \varepsilon_{l}\right)^{2} \geq \frac{1}{4} \sum_{l=1}^{m} \lambda_{l}^{2}\right] \geq \frac{1}{8}, \quad \lambda_{l} \in \mathbb{R}
$$

(see, for example, [7, Lemma 0.2.1] for $\lambda=1 / 4$ ). Thus

$$
\mathrm{P}^{\prime}\left[\omega^{\prime}:\left(\sum_{l=1}^{m} \varepsilon_{l}\left(\omega^{\prime}\right) \xi_{l}(\omega)\right)^{2} \geq \frac{M}{4}\right] \geq \frac{1}{8}
$$

for all $\omega \in \Omega_{m, M}$. Integrating over $\Omega_{m, M}$ we get

$$
\left(\mathrm{P} \times \mathrm{P}^{\prime}\right)\left[\left(\omega, \omega^{\prime}\right):\left(\sum_{l=1}^{m} \varepsilon_{l}\left(\omega^{\prime}\right) \xi_{l}(\omega)\right)^{2} \geq \frac{M}{4}\right] \geq \frac{\varepsilon_{0}}{8} .
$$

Hence there exists $\omega_{0}^{\prime} \in \Omega^{\prime}$ such that

$$
\mathrm{P}\left[\omega:\left(\sum_{l=1}^{m} \varepsilon_{l}\left(\omega_{0}^{\prime}\right) \xi_{l}(\omega)\right)^{2} \geq \frac{M}{4}\right] \geq \frac{\varepsilon_{0}}{8} .
$$

Since $\varepsilon_{l}\left(\omega_{0}^{\prime}\right)= \pm 1$, for the function $\bar{g}(x)=\sum_{l=1}^{m} \varepsilon_{l}\left(\omega_{0}^{\prime}\right) f_{l}(x)$ we have

$$
|\bar{g}(x)| \leq \bar{f}(x), \quad \mathrm{P}\left[\left|\int_{\mathrm{X}} \bar{g} d \mu\right| \geq \frac{\sqrt{M}}{2}\right] \geq \frac{\varepsilon_{0}}{8} .
$$

Recall that $\varepsilon_{0}>0$ is fixed while $M$ is arbitrary. By the dominated convergence theorem [7, Proposition 7.1.1], the set function $\int_{B} \bar{f} d \mu, B \in \mathcal{B}$, is a stochastic measure. Applying [11, Lemma 1] (or [12, Theorem 1.2]) we reach a contradiction with the boundedness in probability of the set of values of the stochastic measure (see [19, Theorem A] or [7, Theorem B.2.1]). 
We consider the Besov spaces $B_{22}^{\alpha}([c, d])$. Recall that the norm in this classical space for $0<\alpha<1$ may be introduced by

$$
\|g\|_{B_{22}^{\alpha}([c, d])}=\|g\|_{L_{2}([c, d])}+\left(\int_{0}^{d-c}\left(w_{2}(g, r)\right)^{2} r^{-2 \alpha-1} d r\right)^{1 / 2},
$$

where

$$
w_{2}(g, r)=\sup _{0 \leq h \leq r}\left(\int_{c}^{d-h}|g(y+h)-g(y)|^{2} d y\right)^{1 / 2} .
$$

The norm in the Besov space $B_{22}^{\alpha}([c, d])$ is equivalent to the norm in the Slobodetskiu space $W_{2}^{\alpha}$.

For any $j \in \mathbb{R}$ and all $n \geq 0$, put

$$
d_{k n}^{(j)}=j+k 2^{-n}, \quad 0 \leq k \leq 2^{n}, \quad \Delta_{k n}^{(j)}=\left(d_{(k-1) n}^{(j)}, d_{k n}^{(j)}\right], \quad 1 \leq k \leq 2^{n} .
$$

The following estimate is a key tool for the proof of Hölder regularity of the stochastic integral. In our estimates, $C$ will denote a positive constant that may change from one place to another.

Lemma 3.2. Let $\mu$ be defined on the Borel $\sigma$-algebra of $\mathbb{R}, Z$ be an arbitrary set, and $q(z, x): Z \times[j, j+1] \rightarrow \mathbb{R}$ be a function such that for some $1 / 2<\alpha<1$ and for each $z \in Z, q(z, \cdot) \in B_{22}^{\alpha}([j, j+1])$. Then the random function

$$
\eta(z)=\int_{[j, j+1]} q(z, x) d \mu(x), \quad z \in Z,
$$

has a version $\tilde{\eta}(z)$ such that for some constant $C$ (independent of $z, j, \omega$ ) and each $\omega \in \Omega$,

$$
\begin{aligned}
& |\tilde{\eta}(z)| \leq|q(z, j) \mu([j, j+1])| \\
& \quad+C\|q(z, \cdot)\|_{B_{22}^{\alpha}([j, j+1])}\left\{\sum_{n \geq 1} 2^{n(1-2 \alpha)} \sum_{1 \leq k \leq 2^{n}}\left|\mu\left(\Delta_{k n}^{(j)}\right)\right|^{2}\right\}^{1 / 2} .
\end{aligned}
$$

Proof. Consider the functions

$$
q_{n}(z, x)=q(z, j) \mathbf{1}_{\{j\}}(x)+\sum_{1 \leq k \leq 2^{n}} q\left(z, d_{(k-1) n}^{(j)}\right) \mathbf{1}_{\Delta_{k n}^{(j)}}(x), \quad n \geq 0 .
$$

From the properties of Besov spaces it follows that for $\alpha>1 / 2$ we have $B_{22}^{\alpha}([j, j+1]) \subset C([j, j+1])$. The dominated convergence theorem $[7$, Proposition 7.1.1] implies that

$$
\int_{[j, j+1]} q_{n}(z, x) d \mu(x) \stackrel{\mathrm{P}}{\rightarrow} \int_{[j, j+1]} q(z, x) d \mu(x), \quad n \rightarrow \infty,
$$


for each $z$. Therefore

$$
\begin{aligned}
\tilde{\eta}(z)= & \int_{[j, j+1]} q_{0}(z, x) d \mu(x) \\
& +\sum_{n \geq 1}\left(\int_{[j, j+1]} q_{n}(z, x) d \mu(x)-\int_{[j, j+1]} q_{n-1}(z, x) d \mu(x)\right)
\end{aligned}
$$

is a version of $\eta(z)$. Using (3.4) and the Cauchy-Schwarz inequality we obtain, for any $\beta>0$,

$$
\begin{aligned}
& \sum_{n \geq 1}\left|\int_{[j, j+1]} q_{n}(z, x) d \mu(x)-\int_{[j, j+1]} q_{n-1}(z, x) d \mu(x)\right| \\
& \leq \sum_{n \geq 1} \sum_{1 \leq k \leq 2^{n}}\left|q\left(z, d_{(k-1) n}^{(j)}\right)-q\left(z, d_{\left(k^{\prime}-1\right)(n-1)}^{(j)}\right)\right|\left|\mu\left(\Delta_{k n}^{(j)}\right)\right| \\
& \leq\left\{\sum_{n \geq 1} \sum_{1 \leq k \leq 2^{n}} 2^{2 n \beta}\left|q\left(z, d_{(k-1) n}^{(j)}\right)-q\left(z, d_{\left(k^{\prime}-1\right)(n-1)}^{(j)}\right)\right|^{2}\right\}^{1 / 2} \\
& \times\left\{\sum_{n \geq 1} \sum_{1 \leq k \leq 2^{n}} 2^{-2 n \beta}\left|\mu\left(\Delta_{k n}^{(j)}\right)\right|^{2}\right\}^{1 / 2} .
\end{aligned}
$$

(The number $k^{\prime}$ is chosen such that $\Delta_{k n}^{(j)} \subset \Delta_{k^{\prime}(n-1)}^{(j)}$.)

Theorem 1.1 of [6] implies that for some constant $C$ and with $\alpha=\beta+1 / 2$,

$$
\begin{aligned}
&\left\{\sum_{n \geq 1} \sum_{1 \leq k \leq 2^{n}} 2^{2 n \beta}\left|q\left(z, d_{(k-1) n}^{(j)}\right)-q\left(z, d_{\left(k^{\prime}-1\right)(n-1)}^{(j)}\right)\right|^{2}\right\}^{1 / 2} \\
& \leq C\|q(z, \cdot)\|_{B_{22}^{\alpha}([j, j+1])} .
\end{aligned}
$$

Applying (3.5) we obtain (3.3).

Note that by Lemma 3.1 , for $\beta>0$,

$$
\sum_{n \geq 1} \sum_{1 \leq k \leq 2^{n}} 2^{-2 n \beta}\left|\mu\left(\Delta_{k n}^{(j)}\right)\right|^{2}<\infty \quad \text { a.s. }
$$

In the following we will take the version of the parameter integral $\eta(z)$ that satisfies (3.3) (we will call it the regular version).

4. The problem. Consider equation (1.1) in the following mild sense:

$$
\begin{aligned}
u(t, x)= & \int_{\mathbb{R}} p(t, x-y) u_{0}(y) d y+\int_{0}^{t} d s \int_{\mathbb{R}} p(t-s, x-y) f(s, y, u(s, y)) d y \\
& +\int_{\mathbb{R}} d \mu(y) \int_{0}^{t} p(t-s, x-y) \sigma(s, y) d s .
\end{aligned}
$$


Here

$$
p(t, x)=\frac{1}{2 a \sqrt{\pi t}} e^{-|x|^{2} / 4 a^{2} t}
$$

is the Gaussian heat kernel, $u(t, x)=u(t, x, \omega):[0, T] \times \mathbb{R} \times \Omega \rightarrow \mathbb{R}$ is an unknown measurable random function, and $\mu$ is a stochastic measure defined on the Borel $\sigma$-algebra of $\mathbb{R}$. The integrals of random functions with respect to $d s$ and $d y$ are taken for each fixed $\omega \in \Omega$ (some properties of such integrals are studied in [10]).

We make the following assumptions throughout the paper.

Assumption 1. $u_{0}(y)=u_{0}(y, \omega): \mathbb{R} \times \Omega \rightarrow \mathbb{R}$ is measurable and bounded, $\left|u_{0}(y, \omega)\right| \leq C_{u_{0}}(\omega)$.

Assumption 2. $u_{0}(y)$ is Hölder continuous in $y \in \mathbb{R}$,

$$
\left|u_{0}\left(y_{1}\right)-u_{0}\left(y_{2}\right)\right| \leq L_{u_{0}}(\omega)\left|y_{1}-y_{2}\right|^{\beta\left(u_{0}\right)}, \quad \beta\left(u_{0}\right) \geq 1 / 6 .
$$

Assumption 3. $f(s, y, z):[0, T] \times \mathbb{R} \times \mathbb{R} \rightarrow \mathbb{R}$ is measurable and bounded, $|f(s, y, z)| \leq C_{f}$.

Assumption 4. $f(s, y, z)$ is uniformly Lipschitz in $y, z \in \mathbb{R}$,

$$
\left|f\left(s, y_{1}, z_{1}\right)-f\left(s, y_{2}, z_{2}\right)\right| \leq L_{f}\left(\left|y_{1}-y_{2}\right|+\left|z_{1}-z_{2}\right|\right) .
$$

Assumption 5. $\sigma(s, y):[0, T] \times \mathbb{R} \rightarrow \mathbb{R}$ is measurable and bounded, $|\sigma(s, y)| \leq C_{\sigma}$.

Assumption 6. $\sigma(s, y)$ is uniformly Hölder continuous in $y$,

$$
\left|\sigma\left(s, y_{1}\right)-\sigma\left(s, y_{2}\right)\right| \leq L_{\sigma}\left|y_{1}-y_{2}\right|^{\beta(\sigma)}, \quad \beta(\sigma)>1 / 2 .
$$

Note that the constants $L_{f}$ and $L_{\sigma}$ are independent of $s$. Also define

$$
\tilde{\beta}=\min \{2 \beta(\sigma), 3 / 2\} \text {. }
$$

Recall that $\int_{\mathbb{R}} p(t, x) d x=1$.

5. Hölder continuity in $x$. We investigate the regularity of the paths of the stochastic integral from (4.1).

Lemma 5.1. Suppose the function $|y|^{\tau}$ is integrable with respect to $\mu$ on $\mathbb{R}$ for some $\tau>3 / 2$ and Assumptions 1-6 hold. Then, for any fixed $t \in[0, T], K>0$ and $\gamma_{1}<(\tilde{\beta}-1) / 2 \tilde{\beta}$, the stochastic process

$$
\vartheta(x)=\int_{\mathbb{R}} d \mu(y) \int_{0}^{t} p(t-s, x-y) \sigma(s, y) d s, \quad|x| \leq K,
$$

has a version Hölder continuous with exponent $\gamma_{1}$. 
Proof. For fixed $t, x_{1}, x_{2}$ define

$$
\begin{aligned}
g(y) & =\int_{0}^{t} p\left(t-s, x_{1}-y\right) \sigma(s, y) d s-\int_{0}^{t} p\left(t-s, x_{2}-y\right) \sigma(s, y) d s \\
& =\int_{0}^{t} \frac{1}{2 a \sqrt{\pi(t-s)}}\left(e^{-\frac{\left|x_{1}-y\right|^{2}}{4 a^{2}(t-s)}}-e^{-\frac{\left|x_{2}-y\right|^{2}}{4 a^{2}(t-s)}}\right) \sigma(s, y) d s .
\end{aligned}
$$

In Step 1 below, for the difference $\vartheta\left(x_{1}\right)-\vartheta\left(x_{2}\right)$, we estimate the integral $\int_{[j, j+1]} g(y) d \mu(y)$. Here we will apply Lemma 3.2. In Step 2, we consider $\int_{\mathbb{R}} g(y) d \mu(y)$ and apply Lemma 3.1.

SteP 1. We start from the following simple estimate. The inequality $1-e^{-\delta x} \leq \delta x$ for $\delta \geq 0$ yields

$$
\int_{0}^{t} \frac{1}{\sqrt{r}}\left(1-e^{-\delta / r}\right) d r=\int_{0}^{\delta}+\int_{\delta}^{t} \leq \int_{0}^{\delta} \frac{1}{\sqrt{r}} d r+\int_{\delta}^{t} \frac{1}{\sqrt{r}} \frac{\delta}{r} d r \leq 4 \sqrt{\delta} .
$$

Thus, for $\delta_{1}, \delta_{2} \geq 0$, we obtain

$$
\int_{0}^{t} \frac{1}{\sqrt{r}}\left|e^{-\delta_{1} / r}-e^{-\delta_{2} / r}\right| d r \leq \int_{0}^{t} \frac{1}{\sqrt{r}}\left(1-e^{-\left|\delta_{2}-\delta_{1}\right| / r}\right) d r \leq 4 \sqrt{\left|\delta_{2}-\delta_{1}\right|} .
$$

Our first aim is to estimate $\|g\|_{B_{22}^{\alpha}([j, j+1])}$ using the value $\left|x_{2}-x_{1}\right|^{\gamma_{1}}$ for an appropriate constant $\gamma_{1}>0$.

To this end, we use (3.2) and consider

$$
\begin{aligned}
& g(y+h)-g(y) \\
& =C \int_{0}^{t} \frac{1}{\sqrt{t-s}}\left(e^{-\frac{\left|x_{1}-y\right|^{2}}{4 a^{2}(t-s)}}-e^{-\frac{\left|x_{2}-y\right|^{2}}{4 a^{2}(t-s)}}\right)(\sigma(s, y+h)-\sigma(s, y)) d s \\
& \quad+C \int_{0}^{t} \frac{\sigma(s, y+h)}{\sqrt{t-s}}\left(e^{-\frac{\left|x_{1}-y-h\right|^{2}}{4 a^{2}(t-s)}}-e^{-\frac{\left|x_{2}-y-h\right|^{2}}{4 a^{2}(t-s)}}-e^{-\frac{\left|x_{1}-y\right|^{2}}{4 a^{2}(t-s)}}+e^{-\frac{\left|x_{2}-y\right|^{2}}{4 a^{2}(t-s)}}\right) d s \\
& =I_{1}+I_{2} .
\end{aligned}
$$

We have $|x| \leq K$ and $|y| \leq|j|+1$. Assumption 5 and (5.1) for one term of $I_{1}$ lead to the estimate

$$
\begin{aligned}
& \left|\int_{0}^{t} \frac{1}{\sqrt{t-s}}\left(e^{-\frac{\left.\mid x_{1}-y\right)\left.\right|^{2}}{4 a^{2}(t-s)}}-e^{-\frac{\left|x_{2}-y\right|^{2}}{4 a^{2}(t-s)}}\right) \sigma(s, y+h) d s\right| \\
& \leq C_{\sigma} \int_{0}^{t} \frac{1}{\sqrt{t-s}}\left|e^{-\frac{\left|x_{1}-y\right|^{2}}{4 a^{2}(t-s)}}-e^{-\frac{\left|x_{2}-y\right|^{2}}{4 a^{2}(t-s)}}\right| d s
\end{aligned}
$$




$$
\begin{aligned}
& \leq C\left|x_{2}-x_{1}\right|^{1 / 2}\left|x_{1}+x_{2}-2 y\right|^{1 / 2} \\
& \leq C(K+|j|+1)^{1 / 2}\left|x_{2}-x_{1}\right|^{1 / 2} .
\end{aligned}
$$

The second term of $I_{1}$ is similar. Thus we obtain

$$
\left|I_{1}\right| \leq C(K+|j|+1)^{1 / 2}\left|x_{2}-x_{1}\right|^{1 / 2} .
$$

Similarly, we can write $I_{2}$ as a sum of two terms and arrive at the same estimate. This way, we obtain

$$
\begin{aligned}
\left(w_{2}(g, r)\right)^{2} & =\sup _{0 \leq h \leq r} \int_{j}^{j+1-h}|g(y+h)-g(y)|^{2} d y \\
& \leq C(K+|j|+1)\left|x_{2}-x_{1}\right| .
\end{aligned}
$$

Further we consider the estimate with a power of $r$. By Assumption 6 applied to $I_{1}$,

$$
\left|I_{1}\right| \leq C h^{\beta(\sigma)}\left|\int_{0}^{t} \frac{1}{\sqrt{t-s}} d s\right| \leq C h^{\beta(\sigma)}, \quad \int_{j}^{j+1-h} I_{1}^{2} d y \leq C h^{2 \beta(\sigma)} .
$$

As in (5.2), using Assumption 5 and (5.1) for one term of $I_{2}$ we get

$$
\begin{aligned}
& \left|\int_{0}^{t} \frac{1}{\sqrt{t-s}}\left(e^{-\frac{\left|x_{1}-y-h\right|^{2}}{4 a^{2}(t-s)}}-e^{-\frac{\left|x_{1}-y\right|^{2}}{4 a^{2}(t-s)}}\right) \sigma(s, y+h) d s\right| \\
& \leq C h^{1 / 2}\left|2 x_{1}-2 y-h\right|^{1 / 2} \leq C(K+|j|+1)^{1 / 2} h^{1 / 2} .
\end{aligned}
$$

The other term of $I_{2}$ is similar. So, for $\beta(\sigma)>1 / 2$,

$$
\left|I_{2}\right| \leq C(K+|j|+1)^{1 / 2} h^{1 / 2} .
$$

On the other hand, by the boundedness of $\sigma$ together with the Lagrange formula, for $\theta=\min \left\{\left|x_{1}-y-h\right|,\left|x_{1}-y\right|\right\}$ and $v=\theta(2 a \sqrt{t-s})^{-1}$ we have

$$
\begin{aligned}
& \left|\int_{0}^{t} \frac{1}{\sqrt{t-s}}\left(e^{-\frac{\left|x_{1}-y-h\right|^{2}}{4 a^{2}(t-s)}}-e^{-\frac{\left|x_{1}-y\right|^{2}}{4 a^{2}(t-s)}}\right) \sigma(s, y+h) d s\right| \\
& \leq C \int_{0}^{t} \frac{1}{\sqrt{t-s}} e^{-\frac{\theta^{2}}{4 a^{2}(t-s)}}\left|\frac{\left(x_{1}-y-h\right)^{2}}{4 a^{2}(t-s)}-\frac{\left(x_{1}-y\right)^{2}}{4 a^{2}(t-s)}\right| d s \\
& \leq C(K+|j|+1) h \int_{0}^{t} \frac{e^{-\frac{\theta^{2}}{4 a^{2}(t-s)}}}{(t-s)^{3 / 2}} d s \\
& \quad=C(K+|j|+1) h \theta^{-1} \int_{\theta / 2 a \sqrt{t}}^{\infty} e^{-v^{2}} d v \leq C(K+|j|+1) h \theta^{-1} .
\end{aligned}
$$


Note that $\theta \geq\left|y-x_{1}\right| / 2$ for $\left|y-x_{1}\right| \geq 2 \sqrt{h}$. For the integral on this domain, we use the estimate (5.7):

$$
\begin{gathered}
\int_{[j, j+1-h] \cap\left\{\left|y-x_{1}\right| \geq 2 \sqrt{h}\right\}} I_{2}^{2} d y \\
\leq C(K+|j|+1)^{2} h^{2} \int_{\left\{\left|y-x_{1}\right| \geq 2 \sqrt{h}\right\}} \frac{1}{\left|y-x_{1}\right|^{2}} d y=C(K+|j|+1)^{2} h^{3 / 2} .
\end{gathered}
$$

For the other domain of integration, we apply (5.6) to see that

$$
\int_{[j, j+1-h] \cap\left\{\left|y-x_{1}\right| \leq 2 \sqrt{h}\right\}} I_{2}^{2} d y \leq C(K+|j|+1) h^{3 / 2} .
$$

So, from (5.4), (5.8), and (5.9) we find that

$$
\left(w_{2}(g, r)\right)^{2} \leq C(K+|j|+1)^{2} r^{\tilde{\beta}} .
$$

The product of (5.3) to the power $\lambda$, and (5.10) to the power $1-\lambda$, $0<\lambda<1$, now satisfies

$$
\left(w_{2}(g, r)\right)^{2} \leq C(K+|j|+1)^{2} r^{\tilde{\beta}(1-\lambda)}\left|x_{2}-x_{1}\right|^{\lambda} .
$$

Therefore, for $\lambda<1-2 \alpha / \tilde{\beta}$ the integral in (3.2) is finite.

Further, estimates analogous to (5.2) yield

$$
\|g\|_{L_{2}([j, j+1])} \leq C(K+|j|+1)^{1 / 2}\left|x_{2}-x_{1}\right|^{1 / 2} .
$$

Similarly, we obtain

$$
|g(j)| \leq C(K+|j|+1)^{1 / 2}\left|x_{2}-x_{1}\right|^{1 / 2} .
$$

Therefore, for any $0<\gamma_{1}<(\tilde{\beta}-1) / 2 \tilde{\beta}$ there exists $\alpha>1 / 2$ such that

$$
\|g\|_{B_{22}^{\alpha}([j, j+1])} \leq C(K+|j|+1)\left|x_{2}-x_{1}\right|^{\gamma_{1}}
$$

STEP 2. We apply Lemma 3.2 for the parameter $z=\left(x_{1}, x_{2}, t\right)$ and choose the regular version of integrals on $[j, j+1]$. Using (5.11), (5.12) and the Cauchy-Schwarz inequality, we obtain

$$
\begin{aligned}
\left|\vartheta\left(x_{1}\right)-\vartheta\left(x_{2}\right)\right| & =\left|\int_{\mathbb{R}} g(y) d \mu(y)\right| \leq \sum_{j \in \mathbb{Z}}\left|\int_{j}^{j+1} g(y) d \mu(y)\right| \\
\leq & \sum_{j \in \mathbb{Z}}|g(j) \mu([j, j+1])| \\
& +C \sum_{j \in \mathbb{Z}}\|g\|_{B_{22}^{\alpha}([j, j+1])}\left\{\sum_{n \geq 1} 2^{n(1-2 \alpha)} \sum_{1 \leq k \leq 2^{n}}\left|\mu\left(\Delta_{k n}^{(j)}\right)\right|^{2}\right\}^{1 / 2}
\end{aligned}
$$




$$
\begin{aligned}
\leq & C\left|x_{2}-x_{1}\right|^{\gamma_{1}}\left[\sum_{j \in \mathbb{Z}}(K+|j|+1)^{1 / 2}|\mu([j, j+1])|\right. \\
& \left.+\sum_{j \in \mathbb{Z}}(K+|j|+1)\left\{\sum_{n \geq 1} 2^{n(1-2 \alpha)} \sum_{1 \leq k \leq 2^{n}}\left|\mu\left(\Delta_{k n}^{(j)}\right)\right|^{2}\right\}^{1 / 2}\right] \\
\leq & C\left|x_{2}-x_{1}\right|^{\gamma_{1}} \\
& \times\left[\left(\sum_{j \in \mathbb{Z}}(|j|+1)^{2 \rho}(K+|j|+1)(\mu([j, j+1]))^{2}\right)^{1 / 2}\left(\sum_{j \in \mathbb{Z}}(|j|+1)^{-2 \rho}\right)^{1 / 2}\right. \\
& +\left(\sum_{n \geq 1} 2^{n(1-2 \alpha)} \sum_{j \in \mathbb{Z}}(K+|j|+1)^{2}(|j|+1)^{2 \rho} \sum_{1 \leq k \leq 2^{n}}\left|\mu\left(\Delta_{k n}^{(j)}\right)\right|^{2}\right)^{1 / 2} \\
& \left.\times\left(\sum_{j \in \mathbb{Z}}(|j|+1)^{-2 \rho}\right)^{1 / 2}\right] .
\end{aligned}
$$

For any $\rho>1 / 2$, we have $\sum_{j \in \mathbb{Z}}(|j|+1)^{-2 \rho}<\infty$. The sums involving the stochastic measures have the form $\sum_{l=1}^{\infty}\left(\int_{\mathrm{X}} f_{l} d \mu\right)^{2}$, where

$$
\left\{f_{l}(y), l \geq 1\right\}=\left\{(|j|+1)^{\rho}(K+|j|+1)^{1 / 2} \mathbf{1}_{[j, j+1]}(y), j \in \mathbb{Z}\right\}
$$

in the first part, and

$$
\begin{aligned}
& \left\{f_{l}(y), l \geq 1\right\}=\left\{2^{n(1-2 \alpha) / 2}(K+|j|+1)(|j|+1)^{\rho} \mathbf{1}_{\Delta_{k n}^{(j)}}(y),\right. \\
& \left.\quad j \in \mathbb{Z}, n \geq 1,1 \leq k \leq 2^{n}\right\}
\end{aligned}
$$

in the second part of the last sum. Lemma 3.1 together with our integrability condition for $\tau=\rho+1$ finishes the proof.

Note that the estimate $\tilde{\beta} \leq 3 / 2$ implies $\gamma_{1}<1 / 6$.

\section{Hölder continuity in $t$}

Lemma 6.1. Assume that for some $\tau>5 / 2$, the function $|y|^{\tau}$ is integrable with respect to $\mu$ on $\mathbb{R}$ and Assumptions 1-6 hold. Then for any fixed $x \in \mathbb{R}$ and $\gamma_{2}<(\tilde{\beta}-1) / 6 \tilde{\beta}$ the stochastic process

$$
\bar{\vartheta}(t)=\int_{\mathbb{R}} d \mu(y) \int_{0}^{t} p(t-s, x-y) \sigma(s, y) d s, \quad t \in[0, T],
$$

has a version Hölder continuous with exponent $\gamma_{2}$.

Proof. For fixed $x, t_{1}, t_{2}$ define

$$
\bar{g}(y)=\int_{0}^{t_{2}} p\left(t_{2}-s, x-y\right) \sigma(s, y) d s-\int_{0}^{t_{1}} p\left(t_{1}-s, x-y\right) \sigma(s, y) d s .
$$


In Steps 1-3 below, we consider the difference $\bar{\vartheta}\left(t_{2}\right)-\bar{\vartheta}\left(t_{1}\right)$, and estimate different parts of the integral $\int_{[j, j+1]} \bar{g}(y) d \mu(y)$. We will apply Lemma 3.2. In Step 4, we consider $\int_{\mathbb{R}} \bar{g}(y) d \mu(y)$ using Lemma 3.1.

We will estimate $\|\bar{g}\|_{B_{22}^{\alpha}([j, j+1])}$ using the value $\left|t_{2}-t_{1}\right|^{\gamma_{2}}$. In this section it is assumed that $t_{1}<t_{2}$. To estimate (3.2), we will write

$$
\bar{g}(y+h)-\bar{g}(y)=J_{1}-J_{2}, \quad J_{1}=J_{11}+J_{12}, \quad J_{2}=J_{21}+J_{22}=\bar{J}_{21}+\bar{J}_{22}
$$

and consider each of the terms

$$
N\left(J_{m n}\right)=\int_{0}^{1} r^{-2 \alpha-1}\left(\sup _{0 \leq h \leq r} \int_{j}^{j+1-h} J_{m n}^{2} d y\right) d r, \quad 1 / 2<\alpha<1,
$$

separately.

SteP 1. Consider

$$
J_{1}=\int_{t_{1}}^{t_{2}} \frac{e^{-\frac{|x-y-h|^{2}}{4 a^{2}\left(t_{2}-s\right)}}}{2 a \sqrt{\pi\left(t_{2}-s\right)}} \sigma(s, y+h) d s-\int_{t_{1}}^{t_{2}} \frac{e^{-\frac{|x-y|^{2}}{4 a^{2}\left(t_{2}-s\right)}}}{2 a \sqrt{\pi\left(t_{2}-s\right)}} \sigma(s, y) d s .
$$

By Assumption 6, we have

$$
\begin{aligned}
\left|J_{11}\right| & =\left|\int_{t_{1}}^{t_{2}} \frac{e^{-\frac{|x-y-h|^{2}}{4 a^{2}\left(t_{2}-s\right)}}}{2 a \sqrt{\pi\left(t_{2}-s\right)}}(\sigma(s, y+h)-\sigma(s, y)) d s\right| \\
& \leq C h^{\beta(\sigma)} \int_{t_{1}}^{t_{2}} \frac{1}{\sqrt{t_{2}-s}} d s=C h^{\beta(\sigma)} \sqrt{t_{2}-t_{1}} .
\end{aligned}
$$

So, for $1 / 2<\alpha<\beta(\sigma)$,

$$
N\left(J_{11}\right) \leq C\left(t_{2}-t_{1}\right) .
$$

As in (5.7), for the second term of $J_{1}$ for $\zeta=\min \{|x-y-h|,|x-y|\}$ and $v=\zeta^{2}\left(4 a^{2}\left(t_{2}-s\right)\right)^{-1}$ we have

$$
\begin{aligned}
\left|J_{12}\right| & =\left|\int_{t_{1}}^{t_{2}} \frac{1}{2 a \sqrt{\pi\left(t_{2}-s\right)}}\left(e^{-\frac{|x-y-h|^{2}}{4 a^{2}\left(t_{2}-s\right)}}-e^{-\frac{|x-y|^{2}}{4 a^{2}\left(t_{2}-s\right)}}\right) \sigma(s, y) d s\right| \\
& \leq C h(|x|+|y|+1) \int_{t_{1}}^{t_{2}} \frac{e^{-\frac{\zeta^{2}}{4 a^{2}\left(t_{2}-s\right)}}}{\left(t_{2}-s\right)^{3 / 2}} d s \\
& =C h(|x|+|y|+1) \zeta^{-1} \int^{\infty} \frac{e^{-v}}{\sqrt{v}} d v \\
& \leq C h(|x|+|j|+1) \zeta^{-2} \sqrt{t_{2}-t_{1}} .
\end{aligned}
$$


(In the last inequality we have used the fact that $|y| \leq|j|+1$ and $\sqrt{v} \geq$ $\zeta\left(2 a \sqrt{t_{2}-t_{1}}\right)^{-1}$ in the domain of integration.)

We will estimate $\int_{[j, j+1-h]} J_{12}^{2} d y$ on two domains separately. Take any $0<\varphi<1 / 3$. Then for $|y-x| \geq 2 h^{\varphi}$ we have $\zeta \geq|y-x| / 2$. Using (6.2), we get

$$
\begin{aligned}
\int_{[j, j+1-h] \cap\left\{|y-x| \geq 2 h^{\varphi}\right\}} & J_{12}^{2} d y \\
& \leq C\left(t_{2}-t_{1}\right) h^{2}(|x|+|j|+1)^{2} \int_{\left\{|y-x| \geq 2 h^{\varphi}\right\}} \frac{d y}{|y-x|^{4}} \\
& =C(|x|+|j|+1)^{2}\left(t_{2}-t_{1}\right) h^{2-3 \varphi} .
\end{aligned}
$$

Consider the integral of $J_{12}^{2}$ on the other domain. Obviously,

$$
\int_{0}^{t} \frac{1}{\sqrt{r}}\left|e^{-\delta_{2} / r}-e^{-\delta_{1} / r}\right| d r \leq \int_{0}^{t} \frac{1}{\sqrt{r}} d r=2 \sqrt{t} .
$$

Multiplying the power $\lambda$ of this inequality and the power $1-\lambda$ of (5.1), for any $0<\lambda<1$ we get

$$
\int_{0}^{t} \frac{1}{\sqrt{r}}\left|e^{-\delta_{2} / r}-e^{-\delta_{1} / r}\right| d r \leq C\left|\delta_{2}-\delta_{1}\right|^{(1-\lambda) / 2} t^{\lambda / 2} .
$$

This way,

$$
\begin{aligned}
\int_{t_{1}}^{t_{2}} \frac{1}{\sqrt{t_{2}-s}}\left|e^{-\frac{|x-y-h|^{2}}{4 a^{2}\left(t_{2}-s\right)}}-e^{-\frac{|x-y|^{2}}{4 a^{2}\left(t_{2}-s\right)} \mid}\right| d s \\
\leq C\left(\frac{|| x-y-\left.h\right|^{2}-|x-y|^{2} \mid}{4 a^{2}}\right)^{(1-\lambda) / 2}\left(t_{2}-t_{1}\right)^{\lambda / 2} \\
\leq C(|x|+|j|+1) h^{(1-\lambda) / 2}\left(t_{2}-t_{1}\right)^{\lambda / 2} .
\end{aligned}
$$

Combining this with Assumption 5, we get

$$
\int_{[j, j+1-h] \cap\left\{|y-x|<2 h^{\varphi}\right\}} J_{12}^{2} d y \leq C(|x|+|j|+1)^{2} h^{1+\varphi-\lambda}\left(t_{2}-t_{1}\right)^{\lambda} .
$$

From (6.3) and (6.4) it follows that for

$$
2-3 \varphi-2 \alpha>0, \quad 1+\varphi-\lambda-2 \alpha>0
$$

the value $N\left(J_{12}\right)$ is finite. Thus, for any $\lambda<1 / 3$ there exist $1 / 2<\alpha<1$ and $0<\varphi<1 / 3$ such that

$$
N\left(J_{12}\right) \leq C(|x|+|j|+1)^{2}\left(t_{2}-t_{1}\right)^{\lambda} .
$$


Step 2. Consider

$$
\begin{aligned}
J_{2}= & \int_{0}^{t_{1}}\left[\frac{e^{-\frac{|x-y-h|^{2}}{4 a^{2}\left(t_{1}-s\right)}}}{2 a \sqrt{\pi\left(t_{1}-s\right)}}-\frac{e^{-\frac{|x-y-h|^{2}}{4 a^{2}\left(t_{2}-s\right)}}}{2 a \sqrt{\pi\left(t_{2}-s\right)}}\right] \sigma(s, y+h) d s \\
& -\int_{0}^{t_{1}}\left[\frac{e^{-\frac{|x-y|^{2}}{4 a^{2}\left(t_{1}-s\right)}}}{2 a \sqrt{\pi\left(t_{1}-s\right)}}-\frac{e^{-\frac{|x-y|^{2}}{4 a^{2}\left(t_{2}-s\right)}}}{2 a \sqrt{\pi\left(t_{2}-s\right)}}\right] \sigma(s, y) d s=J_{21}+J_{22} .
\end{aligned}
$$

SteP 2.1. We estimate $J_{22}$; the term $J_{21}$ is similar. We have

$$
\begin{aligned}
\left|J_{22}\right| \leq & C \int_{0}^{t_{1}}\left[\left(\frac{1}{\sqrt{t_{1}-s}}-\frac{1}{\sqrt{t_{2}-s}}\right) e^{-\frac{|x-y|^{2}}{4 a^{2}\left(t_{2}-s\right)}}\right. \\
& \left.+\frac{1}{\sqrt{t_{1}-s}}\left(e^{-\frac{|x-y|^{2}}{4 a^{2}\left(t_{2}-s\right)}}-e^{-\frac{|x-y|^{2}}{4 a^{2}\left(t_{1}-s\right)}}\right)\right] d s .
\end{aligned}
$$

For the first term of (6.7), we estimate the exponential by 1 to get

$$
\int_{0}^{t_{1}}\left(\frac{1}{\sqrt{t_{1}-s}}-\frac{1}{\sqrt{t_{2}-s}}\right) e^{-\frac{|x-y|^{2}}{4 a^{2}\left(t_{2}-s\right)}} d s \leq 2 \sqrt{t_{2}-t_{1}} .
$$

The second term of (6.7) will be divided into two parts:

$$
\int_{0}^{t_{1}} \frac{1}{\sqrt{t_{1}-s}}\left(e^{-\frac{|x-y|^{2}}{4 a^{2}\left(t_{2}-s\right)}}-e^{-\frac{|x-y|^{2}}{4 a^{2}\left(t_{1}-s\right)}}\right) d s=\int_{t_{1}-\sqrt[3]{t_{2}-t_{1}}}^{t_{1}}+\int_{0}^{t_{1}-\sqrt[3]{t_{2}-t_{1}}} .
$$

The first term of (6.9) is bounded by

$$
\int_{t_{1}-\sqrt[3]{t_{2}-t_{1}}}^{t_{1}} \frac{1}{\sqrt{t_{1}-s}} d s=2 \sqrt[6]{t_{2}-t_{1}}
$$

In the second term of (6.9), we apply the inequality $e^{-z_{1}}-e^{-z_{2}} \leq z_{2}-z_{1}$ for $0 \leq z_{1} \leq z_{2}$ to obtain

$$
\begin{gathered}
\int_{0}^{t_{1}-\sqrt[3]{t_{2}-t_{1}}} \frac{1}{\sqrt{t_{1}-s}}\left(e^{-\frac{|x-y|^{2}}{4 a^{2}\left(t_{2}-s\right)}}-e^{-\frac{|x-y|^{2}}{4 a^{2}\left(t_{1}-s\right)}}\right) d s \\
\leq C|x-y|^{2} \int_{0}^{t_{1}-\sqrt[3]{t_{2}-t_{1}}} \frac{t_{2}-t_{1}}{\left(t_{1}-s\right)^{3 / 2}\left(t_{2}-s\right)} d s \leq C(|x|+|y|)^{2}\left(t_{2}-t_{1}\right)^{1 / 6} .
\end{gathered}
$$

In the second integral of (6.11) we change $t_{1}-s$ and $t_{2}-s$ to $\sqrt[3]{t_{2}-t_{1}}$, so that the integrand increases and we arrive at the desired estimate.

Combining (6.8), (6.10) and (6.11) yields

$$
\left|J_{22}\right| \leq C(|x|+|j|+1)^{2}\left|t_{2}-t_{1}\right|^{1 / 6} .
$$


Analogously we can get the same estimate for $J_{21}$. Therefore,

$$
\sup _{0 \leq h \leq r} \int_{j}^{j+1-h} J_{2}^{2} d y \leq C(|x|+|j|+1)^{4}\left|t_{2}-t_{1}\right|^{1 / 3} .
$$

SteP 2.2. Now we turn to $J_{2}$. Write

$$
\begin{aligned}
J_{2}= & \int_{0}^{t_{1}} \frac{1}{2 a \sqrt{\pi\left(t_{1}-s\right)}}\left(e^{-\frac{|x-y-h|^{2}}{4 a^{2}\left(t_{1}-s\right)}} \sigma(s, y+h)-e^{-\frac{|x-y|^{2}}{4 a^{2}\left(t_{1}-s\right)}} \sigma(s, y)\right) d s \\
& -\int_{0}^{t_{1}} \frac{1}{2 a \sqrt{\pi\left(t_{2}-s\right)}}\left(e^{-\frac{|x-y-h|^{2}}{4 a^{2}\left(t_{2}-s\right)}} \sigma(s, y+h)-e^{-\frac{|x-y|^{2}}{4 a^{2}\left(t_{2}-s\right)}} \sigma(s, y)\right) d s \\
= & \bar{J}_{21}+\bar{J}_{22},
\end{aligned}
$$

with

$$
\begin{aligned}
\left|\bar{J}_{21}\right| \leq & \int_{0}^{t_{1}} \frac{1}{2 a \sqrt{\pi\left(t_{1}-s\right)}} e^{-\frac{|x-y-h|^{2}}{4 a^{2}\left(t_{1}-s\right)}}|\sigma(s, y+h)-\sigma(s, y)| d s \\
& +\left|\int_{0}^{t_{1}} \frac{1}{2 a \sqrt{\pi\left(t_{1}-s\right)}}\left(e^{-\frac{|x-y-h|^{2}}{4 a^{2}\left(t_{1}-s\right)}}-e^{-\frac{|x-y|^{2}}{4 a^{2}\left(t_{1}-s\right)}}\right) \sigma(s, y) d s\right| \\
= & \bar{J}_{211}+\bar{J}_{212} .
\end{aligned}
$$

By Assumption 6, we have

$$
\bar{J}_{211} \leq C L_{\sigma} h^{\beta(\sigma)} \int_{0}^{t_{1}} \frac{1}{\sqrt{t_{1}-s}} d s \leq C h^{\beta(\sigma)}, \quad \int_{j}^{j+1-h} \bar{J}_{211}^{2} d y \leq C h^{2 \beta(\sigma)} .
$$

Along the lines leading from (5.5) to (5.9), we get

$$
\int_{j}^{j+1-h} \bar{J}_{212}^{2} d y \leq C(|x|+|j|+1)^{2} h^{3 / 2} .
$$

Now (6.14) and (6.15) show

$$
\sup _{0 \leq h \leq r} \int_{j}^{j+1-h} \bar{J}_{21}^{2} d y \leq C(|x|+|j|+1)^{2} r^{\tilde{\beta}}
$$

Concerning $\bar{J}_{22}$, for $t_{1}<t_{2}$ we have

$$
\left|\bar{J}_{22}\right| \leq C \int_{0}^{t_{2}} \frac{1}{\sqrt{t_{2}-s}}\left|e^{-\frac{|x-y-h|^{2}}{4 a^{2}\left(t_{2}-s\right)}} \sigma(s, y+h)-e^{-\frac{|x-y|^{2}}{4 a^{2}\left(t_{2}-s\right)}} \sigma(s, y)\right| d s .
$$

We may proceed analogously to the estimate of $\bar{J}_{21}$ and obtain (6.16) for $\bar{J}_{22}$. Consequently, 


$$
\sup _{0 \leq h \leq r} \int_{j}^{j+1-h} J_{2}^{2} d y \leq C(|x|+|j|+1)^{2} r^{\tilde{\beta}}
$$

Raising (6.17) to the power $1-\lambda$ and (6.13) to the power $\lambda$, we conclude that

$$
\sup _{0 \leq h \leq r} \int_{j}^{j+1-h} J_{2}^{2} d y \leq C(|x|+|j|+1)^{2+2 \lambda}\left|t_{1}-t_{2}\right|^{\lambda / 3} r^{\tilde{\beta}(1-\lambda)} .
$$

Therefore, for any $\lambda<1-1 / \tilde{\beta}$ there exists $\alpha>1 / 2$ such that

$$
N\left(J_{2}\right) \leq C(|x|+|j|+1)^{2+2 \lambda}\left|t_{1}-t_{2}\right|^{\lambda / 3} .
$$

Recall that we have larger Hölder exponents in (6.1) and (6.5).

Step 3. Consider

$$
\begin{aligned}
|\bar{g}(y)| \leq & \left|\int_{0}^{t_{1}}\left(p\left(t_{1}-s, x-y\right)-p\left(t_{2}-s, x-y\right)\right) \sigma(s, y) d s\right| \\
& +\left|\int_{t_{1}}^{t_{2}} p\left(t_{2}-s, x-y\right) \sigma(s, y) d s\right| .
\end{aligned}
$$

The first integral in (6.19) equals $C J_{22}$, and we can use estimate (6.12). For the second summand of (6.19) we have

$$
\left|\int_{t_{1}}^{t_{2}} p\left(t_{2}-s, x-y\right) \sigma(s, y) d s\right| \leq C \int_{t_{1}}^{t_{2}} \frac{1}{\sqrt{t_{2}-s}} d s=C \sqrt{t_{2}-t_{1}} .
$$

Therefore

$$
\begin{gathered}
|\bar{g}(j)| \leq C(|x|+|j|+1)^{2}\left|t_{2}-t_{1}\right|^{1 / 6}, \\
\|\bar{g}(y)\|_{L_{2}([j, j+1])} \leq C(|x|+|j|+1)^{2}\left|t_{2}-t_{1}\right|^{1 / 6},
\end{gathered}
$$

completing the estimate of the terms of (3.3).

Therefore, for any $0<\gamma_{2}<(\tilde{\beta}-1) / 6 \tilde{\beta}$ there exists $\alpha>1 / 2$ such that

$$
\|\bar{g}\|_{B_{22}^{\alpha}([j, j+1])} \leq C(|x|+|j|+1)^{2}\left|t_{2}-t_{1}\right|^{\gamma_{2}} .
$$

STEP 4. Recall Step 2 of the proof of Lemma 5.1 for the parameter $z=\left(x, t_{1}, t_{2}\right)$ and the function $\bar{g}$. We use (6.20) and (6.21) instead of (5.11) and (5.12), so we get $\tau=\rho+2>5 / 2$.

Note that from $\tilde{\beta} \leq 3 / 2$ it follows that $\gamma_{2}<1 / 18$.

\section{Solution to the equation}

Theorem. Suppose Assumptions 1-6 hold.

(i) Equation (4.1) has a solution $u(t, x)$. If $v(t, x)$ is another solution, then for each $t$ and $x, u(t, x)=v(t, x)$ a.s. 
(ii) Suppose the function $|y|^{\tau}$ is integrable with respect to $\mu$ on $\mathbb{R}$ for some $\tau>3 / 2$. Then, for any fixed $t \in[0, T], K>0$, and $\gamma_{1}<$ $(\tilde{\beta}-1) / 2 \tilde{\beta}$, the stochastic process $u(t, x), x \in[-K, K]$, has a version Hölder continuous with exponent $\gamma_{1}$.

(iii) Suppose the function $|y|^{\tau}$ is integrable with respect to $\mu$ on $\mathbb{R}$ for some $\tau>5 / 2$. Then for any fixed $\delta>0, K>0, \gamma_{2}<(\tilde{\beta}-1) / 6 \tilde{\beta}$, and $\gamma_{1}<(\tilde{\beta}-1) / 2 \tilde{\beta}$, the stochastic function $u(t, x)$ has a version $\tilde{u}(t, x)$ such that for some $L_{\tilde{u}}(\omega)>0$,

$$
\begin{aligned}
\left|\tilde{u}\left(t_{1}, x_{1}\right)-\tilde{u}\left(t_{2}, x_{2}\right)\right| \leq L_{\tilde{u}}\left(\left|t_{1}-t_{2}\right|^{\gamma_{2}}+\left|x_{1}-x_{2}\right|^{\gamma_{1}}\right), & \\
& t \in[\delta, T], x \in[-K, K] .
\end{aligned}
$$

Proof. Consider the following standard iteration process. Take $u^{(0)}(t, x)$ $=0$ and set

$$
\begin{aligned}
u^{(n+1)}(t, x)= & \int_{\mathbb{R}} p(t, x-y) u_{0}(y) d y \\
& +\int_{0}^{t} d s \int_{\mathbb{R}} p(t-s, x-y) f\left(s, y, u^{(n)}(s, y)\right) d y \\
& +\int_{\mathbb{R}} d \mu(y) \int_{0}^{t} p(t-s, x-y) \sigma(s, y) d s, \quad n \geq 0 .
\end{aligned}
$$

The measurability of the first and second summands of (7.1) follows from the Fubini theorem. The third summand may be rewritten as the limit in probability of the integrals of the simple functions

$$
\int_{\mathbb{R}} h_{i}(x, t, y) d \mu(y), \quad i \rightarrow \infty, \quad h_{i}(x, t, y)=\sum_{k=1}^{l^{(i)}} c_{k}^{(i)}(x, t) \mathbf{1}_{A_{k}^{(i)}}(y) .
$$

By $[10$, Lemma 2], the limit in probability of measurable processes is again measurable.

Further, for all $n, t, x$ we take the same version of the stochastic integral, so the estimates below hold for all $\omega \in \Omega$. By Assumption 4, we get

$$
\begin{aligned}
& \left|u^{(n+1)}(t, x)-u^{(n)}(t, x)\right| \\
& \quad \leq L_{f} \int_{0}^{t} d s \int_{\mathbb{R}} p(t-s, x-y)\left|u^{(n)}(s, y)-u^{(n-1)}(s, y)\right| d y, \quad n \geq 2 .
\end{aligned}
$$

By the boundedness of $f$, we obtain

$$
\left|u^{(2)}(t, x)-u^{(1)}(t, x)\right| \leq 2 C_{f} \int_{0}^{t} d s \int_{\mathbb{R}} p(t-s, x-y) d y=2 C_{f} t .
$$


Set

$$
g_{n}(t)=\sup _{x \in \mathbb{R}}\left|u^{(n+1)}(t, x)-u^{(n)}(t, x)\right|, \quad n \geq 1 .
$$

Then from (7.2) we obtain

$$
g_{n}(t) \leq L_{f} \int_{0}^{t} g_{n-1}(s) d s .
$$

By induction,

$$
g_{n}(t) \leq 2 C_{f} L_{f}^{n} \frac{t^{n+1}}{(n+1) !},
$$

hence the series $\sum_{n=0}^{\infty} g_{n}(t)$ converges uniformly on $[0, T]$. We define $u(t, x):=\lim _{n \rightarrow \infty} u^{(n)}(t, x)$. Taking the limit in (7.1) as $n \rightarrow \infty$, we arrive at (4.1).

Note that the paths of the right hand side of (4.1) are continuous in $t, x$ for the regular version of stochastic integral, and $u$ is continuous in probability. We can take the continuous version of $u$ and exclude $\omega$ such that (4.1) fails for some rational $x, t$. Then the estimates below hold for all $\omega$ from a set of probability 1 .

If $u(t, x)$ and $v(t, x)$ are both solutions to (4.1), then

$$
u(t, x)-v(t, x)=\int_{0}^{t} d s \int_{\mathbb{R}} p(t-s, x-y)[f(s, y, u(s, y))-f(s, y, v(s, y))] d y .
$$

Applying Assumption 4, we may use the arguments leading from (7.2) to $(7.3)$ for

$$
g(t)=\sup _{x \in \mathbb{R}}|u(t, x)-v(t, x)|
$$

to get

$$
g(t) \leq 2 C_{f} L_{f}^{n} \frac{t^{n+1}}{(n+1) !} \leq 2 C_{f} L_{f}^{n} \frac{T^{n+1}}{(n+1) !} .
$$

Consequently, $g=0$ and the solution is unique. Part (i) of the theorem is proved.

We now investigate the Hölder continuity in $x$ of the solution on bounded intervals of $\mathbb{R}$.

We claim that for each $n \geq 0$ there is some $L_{u^{(n)}}(t)>0$ such that

$$
\left|u^{(n)}\left(t, x_{1}\right)-u^{(n)}\left(t, x_{2}\right)\right| \leq L_{u^{(n)}}(t)\left|x_{1}-x_{2}\right|^{\gamma_{1}} .
$$

We have $L_{u^{(0)}}=0$. We will proceed by induction. 
Applying (7.1), Lemma 5.1, the change of variables $y \mapsto y+x_{2}-x_{1}$ in the integrals with respect to $d y$ involving $x_{2}$, and our Assumptions, we have

$$
\begin{aligned}
& \left|u^{(n+1)}\left(t, x_{1}\right)-u^{(n+1)}\left(t, x_{2}\right)\right| \\
& \leq \int_{\mathbb{R}} p\left(t, x_{1}-y\right)\left|u_{0}(y)-u_{0}\left(y+x_{2}-x_{1}\right)\right| d y \\
& \quad+\int_{0}^{t} d s \int_{\mathbb{R}} p\left(t-s, x_{1}-y\right) \\
& \quad \times\left|f\left(s, y, u^{(n)}(s, y)\right)-f\left(s, y+x_{2}-x_{1}, u^{(n)}\left(s, y+x_{2}-x_{1}\right)\right)\right| d y \\
& \quad+C\left|x_{1}-x_{2}\right|^{\gamma_{1}} \\
& \leq L_{u_{0}}\left|x_{1}-x_{2}\right|^{\beta\left(u_{0}\right)} \\
& \quad+\int_{0}^{t} d s \int_{\mathbb{R}} p\left(t-s, x_{1}-y\right) L_{f}\left(\left|x_{1}-x_{2}\right|+L_{u^{(n)}}(s)\left|x_{1}-x_{2}\right|^{\gamma_{1}}\right) d y \\
& \quad+C\left|x_{1}-x_{2}\right|^{\gamma_{1}} .
\end{aligned}
$$

Thus,

$$
L_{u^{(n+1)}}(t) \leq L+L \int_{0}^{t} L_{u^{(n)}}(s) d s
$$

for some constant $L$, so the claim is true. Inductively, we obtain the finite upper bound

$$
L_{u^{(n)}}(t) \leq L e^{L t} \leq L e^{L T},
$$

which proves the Hölder continuity in $x$. Thus we get part (ii) of the theorem. Note that $L$ is independent of $t$.

Further, for fixed $x$, we prove the Hölder continuity in $t$ of the solution $u(t, x)$ on intervals $[\delta, T], \delta>0$. For $\delta \leq t_{1}<t_{2} \leq T$ and the regular version of $u(\cdot, x)$, using Lemma 6.1 , we have

$$
\begin{aligned}
\left|u\left(t_{2}, x\right)-u\left(t_{1}, x\right)\right| \leq & \int_{\mathbb{R}}\left|p\left(t_{2}, x-y\right)-p\left(t_{1}, x-y\right)\right|\left|u_{0}(y)\right| d y \\
& +\mid \int_{0}^{t_{2}} d s \int_{\mathbb{R}} p\left(t_{2}-s, x-y\right) f(s, y, u(s, y)) d y \\
& -\int_{0}^{t_{1}} d s \int_{\mathbb{R}} p\left(t_{1}-s, x-y\right) f(s, y, u(s, y)) d y|+C| t_{1}-\left.t_{2}\right|^{\gamma_{2}} \\
= & Y_{1}+Y_{2}+C\left|t_{1}-t_{2}\right|^{\gamma_{2}} .
\end{aligned}
$$

For $Y_{1}$, Assumption 1 shows 


$$
\begin{aligned}
Y_{1} & \leq C \int_{\mathbb{R}}\left|\frac{1}{\sqrt{t_{2}}} e^{-\frac{(x-y)^{2}}{4 a^{2} t_{2}}}-\frac{1}{\sqrt{t_{1}}} e^{-\frac{(x-y)^{2}}{4 a^{2} t_{1}}}\right| d y \\
& \leq C \int_{\mathbb{R}}\left|\frac{1}{\sqrt{t_{2}}}-\frac{1}{\sqrt{t_{1}}}\right| e^{-\frac{(x-y)^{2}}{4 a^{2} t_{1}}} d y+C \int_{\mathbb{R}} \frac{1}{\sqrt{t_{2}}}\left|e^{-\frac{(x-y)^{2}}{4 a^{2} t_{2}}}-e^{-\frac{(x-y)^{2}}{4 a^{2} t_{1}}}\right| d y \\
& =C\left(\frac{1}{\sqrt{t_{1}}}-\frac{1}{\sqrt{t_{2}}}\right) \sqrt{t_{1}}+C \frac{1}{\sqrt{t_{2}}}\left(\sqrt{t_{2}}-\sqrt{t_{1}}\right) \leq C \delta^{-1}\left(t_{2}-t_{1}\right) .
\end{aligned}
$$

Now we estimate $Y_{2}$ in the following way:

$$
\begin{aligned}
Y_{2} \leq & \int_{t_{1}}^{t_{2}} d s \int_{\mathbb{R}} p\left(t_{2}-s, x-y\right)|f(s, y, u(s, y))| d y \\
& \quad+\int_{0}^{t_{1}} d s \int_{\mathbb{R}}\left|p\left(t_{2}-s, x-y\right)-p\left(t_{1}-s, x-y\right)\right||f(s, y, u(s, y))| d y \\
& =Y_{21}+Y_{22} .
\end{aligned}
$$

By boundedness of $f, Y_{21} \leq C_{f}\left(t_{2}-t_{1}\right)$. Using the equality $\int_{\mathbb{R}} e^{-z^{2} / b^{2}} d z=C b$ and some simple calculations, we obtain

$$
\begin{aligned}
Y_{22} \leq & C \int_{0}^{t_{1}} d s \int_{\mathbb{R}} \mid \frac{1}{\sqrt{t_{2}-s}} e^{-\frac{(x-y)^{2}}{4 a^{2}\left(t_{2}-s\right)}}-\frac{1}{\sqrt{t_{1}-s}} e^{-\frac{(x-y)^{2}}{4 a^{2}\left(t_{1}-s\right)} \mid d y} \\
\leq & C \int_{0}^{t_{1}} d s \int_{\mathbb{R}}\left|\frac{1}{\sqrt{t_{2}-s}}-\frac{1}{\sqrt{t_{1}-s}}\right| e^{-\frac{(x-y)^{2}}{4 a^{2}\left(t_{2}-s\right)}} d y \\
& +C \int_{0}^{t_{1}} d s \int_{\mathbb{R}} \frac{1}{\sqrt{t_{1}-s}}\left|e^{-\frac{(x-y)^{2}}{4 a^{2}\left(t_{2}-s\right)}}-e^{-\frac{(x-y)^{2}}{4 a^{2}\left(t_{1}-s\right)}}\right| d y=C \sqrt{t_{2}-t_{1}} .
\end{aligned}
$$

For a given interval $[-K, K]$, the constant $C$ is independent of $x$.

Therefore, under the assumptions of (iii), we can find a version $\tilde{u}^{(x)}$ that is Hölder continuous in $x$ for fixed $t$, and a version $\tilde{u}^{(t)}$ that is Hölder continuous in $t$ for fixed $x$. We exclude all $\omega \in \Omega$ such that $\tilde{u}^{(x)}(t, x) \neq$ $\tilde{u}^{(t)}(t, x)$ for at least one pair of rational $(t, x) \in[\delta, T] \times[-K, K]$. For other $\omega$, we put $\tilde{u}=\tilde{u}^{(x)}=\tilde{u}^{(x)}$ for rational $(t, x)$ and define $\tilde{u}$ on $[\delta, T] \times[-K, K]$ by continuity. Thus we get a version $\tilde{u}(t, x)$ that is Hölder continuous in $x$ and $t$.

We can compare our values of $\gamma_{1}$ and $\gamma_{2}$ with the results obtained for SPDEs with Gaussian noise. In [5], for a class of parabolic SPDEs with Banach space valued noise, it is proved that the solution is Hölder continuous in time with exponent $\alpha$ and Hölder continuous in space with exponent $\beta$, provided that $2 \alpha+\beta<1 / 2$. For the equation studied in $[21$, 
Chapter 3], Corollary 3.4 of [21] states Hölder continuity with any exponent less than $1 / 4$.

Acknowledgements. The paper was prepared during the author's visit to Jena University. The author wishes to thank Prof. M. Zähle for fruitful discussions, and the hospitality of Jena University is gratefully acknowledged.

This work was supported by Alexander von Humboldt Foundation, grant no. UKR/1074615.

\section{References}

[1] S. Albeverio, J.-L. Wu and T.-S. Zhang, Parabolic SPDEs driven by Poisson white noise, Stochastic Process. Appl. 74 (1998), 21-36.

[2] G. P. Curbera and O. Delgado, Optimal domains for $L_{0}$-valued operators via stochastic measures, Positivity 11 (2007), 399-416.

[3] R. C. Dalang and M. Sanz-Solé, Regularity of the sample paths of a class of secondorder spde's, J. Funct. Anal. 227 (2005), 304-337.

[4] G. Da Prato and J. Zabczyk, Stochastic Equations in Infinite Dimensions, Encyclopedia Math. Appl. 44, Cambridge Univ. Press, Cambridge, 1992.

[5] J. Dettweiler, L. Weis, and J. van Neerven, Space-time regularity of solutions of the parabolic stochastic Cauchy problem, Stochastic Anal. Appl. 24 (2006), 843-869.

[6] A. Kamont, A discrete characterization of Besov spaces, Approx. Theory Appl. (N.S.) 13 (1997), 63-77.

[7] S. Kwapień and W. A. Woyczyński, Random Series and Stochastic Integrals: Single and Multiple, Birkhäuser, Boston, 1992.

[8] J. Memin, Y. Mishura, and E. Valkeila, Inequalities for the moments of Wiener integrals with respect to a fractional Brownian motion, Statist. Probab. Lett. 51 (2001), 197-206.

[9] J. M. A. M. van Neerven, M. C. Veraar, and L. Weis, Stochastic evolution equations in UMD Banach spaces, J. Funct. Anal. 255 (2008), 940-993.

[10] V. Radchenko, On a definition of the integral of a random function, Theory Probab. Appl. 41 (1996), 597-601.

[11] - Convergence of integrals of unbounded real functions with respect to random measures, ibid. 42 (1997), 310-314.

[12] - Integrals with respect to general stochastic measures, Institute of Mathematics, Kyiv, 1999 (in Russian).

[13] - , Parameter-dependent integrals with general random measures, Theory Probab. Math. Statist. 75 (2006), 161-165.

[14] —, Besov regularity of stochastic measures, Statist. Probab. Lett. 77 (2007), 822825.

[15] - Heat equation and wave equation with general stochastic measures, Ukraïn. Mat. Zh. 60 (2008), 1675-1685.

[16] S. Rolewicz, Metric Linear Spaces, Monografie Mat. 56, PWN-Polish Sci. Publ., Warszawa, 1972.

[17] C. Ryll-Nardzewski and W. A. Woyczyński, Bounded multiplier convergence in measure of random vector series, Proc. Amer. Math. Soc. 53 (1975), 96-98. 
[18] M. Sanz-Solé and M. Sarrà, Hölder continuity for the stochastic heat equation with spatially correlated noise, in: Progr. Probab. 52, Birkhäuser, Basel, 2002, 259-268.

[19] M. Talagrand, Les mesures vectorielles à valeurs dans $L_{0}$ sont bornées, Ann. Sci. École Norm. Sup. (4) 14 (1981), 445-452.

[20] Ph. Turpin, Convexités dans les espaces vectoriels topologiques généraux, Dissertationes Math. 131 (1976).

[21] J. B. Walsh, An introduction to stochastic partial differential equations, in: Lecture Notes in Math. 1180, Springer, Berlin, 1986, 265-439.

Department of Mathematical Analysis

Faculty of Mechanics and Mathematics

Kyiv National Taras Shevchenko University

Volodymyrska St. 64

Kyiv 01033, Ukraine

E-mail: vradchenko@univ.kiev.ua

Received September 7, 2008

Revised version March 9, 2009 